\title{
Efficacy and safety outcomes of robotic radical hysterectomy in Chinese older women with cervical cancer compared with laparoscopic radical hysterectomy
}

Cheng Luo ${ }^{1+} \mathbb{D}$, Mei Liü ${ }^{2+}$ and Xiuli Li $i^{*}$

\begin{abstract}
Background: Recently, as a complex integrating a number of modern high-tech means, robotic surgery system is a well-deserved revolutionary tool in globally minimally invasive surgical field. For the first time in China, the objective of this study was to evaluate the efficacy and safety outcomes of robotic radical hysterectomy (RRH) in Chinese older women with cervical cancer compared with laparoscopic radical hysterectomy $(\mathrm{LRH})$.

Methods: In this prospective, randomized and double-blinded study, 60 Chinese older women with cervical cancer were evenly divided to accept the RRH or LRH. Follow-up period lasted for 24 months.

Results: Median age for the entire cohort was 65 (range: 61-69) years. There was no difference in International Federation of Gynecology and Obstetrics (FIGO) stages and cell types between two groups ( $p>0.05$ for all). Uterine size, tumor size, vaginal length and numbers of left and right pelvic lymph nodes did not differ between two groups ( $p>0.05$ for all). No difference was observed in numbers of left and right lymph node metastasis $(p>0.05$ for all). All patients had negative margins without conversion to laparotomy. There were significantly less postoperative complications in the RRH group than in the LRH group $(p<0.05)$. Shorter indwelling time of bladder and drain catheters was observed in the RRH group than in the LRH group ( $p<0.05$ for all). Length of postoperative hospital stay in the RRH group was significantly shorter compared with that in the LRH group $(p<0.05)$. Patients in two groups similarly experienced the recurrence and death ( $p>0.05$ for all).

Conclusions: This study demonstrated that RRH provided additional benefits for Chinese older women with cervical cancer because of less complications and faster recovery compared with LRH. Meanwhile, this study supported an equivalence of surgical qualities and survival outcomes of RRH to LRH. Robotics-assisted surgical method is effective, safe and feasible for Chinese older women with cervical cancer.
\end{abstract}

Keywords: Cervical cancer, Chinese older women, Laparoscopic radical hysterectomy, Robotic radical hysterectomy

\section{Background}

Recently, as a complex integrating a number of modern high-tech means, robotic surgery system is a welldeserved revolutionary tool in globally minimally invasive surgical field [1]. It consists of three parts including highresolution imaging device, surgeon operating platform

\footnotetext{
*Correspondence: lixiuli330521@163.com

${ }^{\dagger}$ Equal contributors

'Department of Gynecology, Chinese People's Liberation Army General

Hospital and Hainan Branch, Haitang Bay, Sanya, China

Full list of author information is available at the end of the article
}

and robotic manipulating arm, and adopts the most advanced master-slave remote control operation mode. The superiority provided by this new technology includes 3dimensional magnified field, tremor filtration and wide range of instrument mobility, thus obviously addressing the ergonomic defects of traditional laparoscopic method $[2,3]$. With increased precision and decreased wound, robotic surgery system has the opportunity to improve the efficacy and safety outcomes of surgical operation. Its clinical application has been developing rapidly and widely in various surgical fields. 
Based on Cancer Statistics in China, there were 98,900 (expected incidence) new cases of cervical cancer in 2015, including 23,500 in Chinese older women (over the age of 60). Open radical hysterectomy (ORH) has been a main therapy of cervical cancer in China for a long time [4]. However, traditional surgical method results in uncomfortable symptoms and poor prognosis of patients due to a series of wounds and complications. Laparoscopic radical hysterectomy (LRH) has been confirmed to have the superiority over ORH through making the surgical operation more effective and safe [5, 6]. Several studies have also found the superiority of robotic assistance in solving the obstacles of ORH [7-10]. However, robotic radical hysterectomy (RRH) is an innovative technology with limited application to cervical cancer, and the studies analyzing its application in patients with cervical cancer are still insufficient all over the world [11-13]. Moreover, prospective studies comparing the efficacy and safety outcomes of RRH and LRH are scarce for older women [14, 15]. Meanwhile, robotic surgery system has just been applied in Chinese hospitals recently, and there are hardly any study assessing the clinical value of RRH on Chinese older women with cervical cancer. For the first time in China, the objective of this study was to evaluate the efficacy and safety outcomes of RRH in Chinese older women with cervical cancer compared with LRH.

\section{Methods}

\section{Study patients and procedures}

This study was a prospective, randomized and doubleblinded analysis of Chinese patients older than 60 years with cervical cancer in gynecology department of our hospital conducted from January 2014 to January 2015. There were 60 patients evenly divided to accept the RRH or LRH using random numbers in a randomized block design. Random numbers were generated by Statistic Package for Social Science (SPSS) version 17.0 software (SPSS Inc., Chicago, USA). They all were diagnosed with cervical cancer at International Federation of Gynecology and Obstetrics (FIGO) stage no more than IIB, and had no severe abdominal adhesion and heart, lung, liver and kidney disorders. Patient characteristics and surgical details were recorded by well-trained investigators. Pathological specimens were read by experienced pathologists. Follow-up period lasted for 24 months, and no patient lost from the follow-up. Numbers of recurrence and death were the primary end-points. Total number of postoperative complications and length of postoperative hospital stay were the secondary end-points.

\section{Surgical techniques}

All surgeries were conducted by gynecologists with experience in advanced laparoscopic procedures and made up of the following steps: 1) resection of round ligament of uterus; 2) pelvic lymphadenectomy; 3) ligation and dissection of uterine artery; 4) dissection of ureter; 5) resection of cardinal ligament of uterus and uterosacral ligament; 6) resection of upper vagina; and 7) vaginal cuff closure. RRH was conducted with a da Vinci system (Intuitive Surgical Inc., Sunnyvale, CA, USA). Five trocars were applied as follows: one 12-mm camera trocar placed via the open-Hasson technique, two 8-mm ancillary robotic trocars placed bilaterally (approximately 8-10 $\mathrm{cm}$ away from the camera port and 30 degrees below it to avoid the collisions between the robotic arms), and two assistant trocars. LRH was conducted as laparoscopically assisted operation with four trocars including one $12-\mathrm{mm}$ camera trocar, two operating trocars and one assistant trocar. Nerve sparing technique was not conducted in all patients. After surgical operation, all patients underwent the paclitaxel plus cisplatin chemotherapy (TP regimen) and pelvic external radiotherapy.

\section{Statistical analyses}

Statistical analyses were conducted using SPSS version 17.0 software (SPSS Inc., Chicago, IL, USA). Continuous variables with normal or skewed distribution were shown as mean (standard deviation) or median (interquartile range), and compared by Student's t-test or Mann-Whitney U test, respectively. Categorical variables were shown as number (percentage), and compared by Chi-square test. Two-tailed $p$-values less than 0.05 were regarded as statistically significant.

\section{Results}

Median age for the entire cohort was 65 (range: 61-69) years. Patient characteristics and pathological findings are shown in Table 1. Two surgical groups were similar with respect to age and history of previous pelvic surgeries $(p>0.05$ for all). There was no difference in FIGO stages and cell types between two surgical groups $(p>0.05$ for all). Uterine size, tumor size, vaginal length and numbers of left and right pelvic lymph nodes did not differ between two surgical groups ( $p>0.05$ for all). Numbers of left and right lymph node metastasis had no difference between two surgical groups $(p>0.05$ for all). All patients belong to two surgical groups had negative margins.

All patients were completed through RRH or LRH without conversion to laparotomy. Postoperative complications and survival outcomes are shown in Table 2. There were significantly less total number of postoperative complications in the RRH group than in the LRH group $(p<0.05)$. No significant difference between two surgical groups was seen in postoperative complications including febrile morbidity, port site cellulitis/hernia/dehiscence, urinary tract infection and ureteral injury $(p>0.05$ for all). 
Table 1 Patient characteristics and pathological findings

\begin{tabular}{|c|c|c|c|}
\hline Characteristics & $\begin{array}{l}\text { RRH group } \\
(n=30)\end{array}$ & $\begin{array}{l}\text { LRH group } \\
(n=30)\end{array}$ & $P$ value \\
\hline $\mathrm{Age}^{\mathrm{a}}, \mathrm{yr}$ & $65(62-67)$ & $64(62-66)$ & 0.404 \\
\hline History of previous abdominal surgeries, $n(\%)$ & $12(40.0)$ & 15(50.0) & 0.436 \\
\hline FIGO stages, $n(\%)$ & & & 0.532 \\
\hline IA & $5(16.7)$ & $7(23.3)$ & \\
\hline IB1 & 16(53.3) & 14(46.7) & \\
\hline IB2 & $1(3.3)$ & $3(10.0)$ & \\
\hline$\| \mathrm{A}$ & $4(13.3)$ & $4(13.3)$ & \\
\hline$\| \mathrm{B}$ & $4(13.3)$ & $2(6.7)$ & \\
\hline Cell types, $n(\%)$ & & & 0.380 \\
\hline Squamous & 18(60.0) & $21(70.0)$ & \\
\hline Adenoid & 10(33.3) & $8(26.7)$ & \\
\hline Neuroendocrine & $2(6.7)$ & 1(3.3) & \\
\hline Uterine size ${ }^{a}, \mathrm{~cm}^{3}$ & 153.13(120.00-240.75) & $191.29(147.09-223.62)$ & 0.352 \\
\hline Tumor size ${ }^{\mathrm{a}}, \mathrm{cm}^{3}$ & $4.95(1.43-9.54)$ & $4.76(2.32-11.9)$ & 0.767 \\
\hline Vaginal length ${ }^{\mathrm{a}}, \mathrm{cm}$ & $1.50(1.00-2.30)$ & $1.35(1.00-2.00)$ & 0.337 \\
\hline Left pelvic lymph nodes,$n$ & $9.0(7.0-14.0)$ & $8.5(6.0-13.0)$ & 0.727 \\
\hline Right pelvic lymph nodes ${ }^{a}, n$ & 10.5(7.0-12.0) & 10.0(7.0-11.0) & 0.541 \\
\hline Left pelvic lymph node metastasis ${ }^{a}, n$ & $0(0-0)$ & $0(0-1.0)$ & 0.549 \\
\hline Right pelvic lymph node metastasis ${ }^{a}, n$ & $0(0-0)$ & $0(0-0.5)$ & 0.869 \\
\hline
\end{tabular}

Notes: ${ }^{a}$ median (interquartile range). Abbreviations: $R R H$ robotic radical hysterectomy, $L R H$ laparoscopic radical hysterectomy, FIGO International Federation of Gynecology and Obstetrics

Two surgical groups had no postoperative complication including urinary retention, bowel injury/obstruction, pulmonary embolism and deep vein thrombosis. There was no postoperative complication of vaginal vault, such as bleeding, infection, dehiscence and leakage in two surgical groups. No postoperative complication including

Table 2 Postoperative complications and survival outcomes

\begin{tabular}{|c|c|c|c|}
\hline Characteristics & RRH group & LRH group & $P$ value \\
\hline $\begin{array}{l}\text { Total number of Postoperative } \\
\text { complications, } n(\%)\end{array}$ & $4(6.7)$ & $11(18.3)$ & 0.037 \\
\hline Febrile morbidity, n (\%) & $2(6.7)$ & $5(16.7)$ & 0.421 \\
\hline $\begin{array}{l}\text { Port site cellulitis/hernia/dehiscence, } \\
n(\%)\end{array}$ & $1(3.3)$ & $3(10.0)$ & 0.605 \\
\hline Urinary tract infection, $n(\%)$ & $1(3.3)$ & $2(6.7)$ & 1.000 \\
\hline Ureteral injury, n (\%) & $0(0)$ & $1(3.3)$ & 1.000 \\
\hline $\begin{array}{l}\text { Indwelling bladder catheter } \\
\text { time }^{a}, d\end{array}$ & $6(5-11)$ & $7(6-11)$ & 0.043 \\
\hline $\begin{array}{l}\text { Indwelling drain catheter } \\
\text { time }^{a}, d\end{array}$ & $29(23-36)$ & $32(28-38)$ & 0.038 \\
\hline $\begin{array}{l}\text { Length of postoperative } \\
\text { hospital stay }, d\end{array}$ & 13(10-15) & $15(11-17)$ & 0.042 \\
\hline Recurrence, $n(\%)$ & $2(6.7)$ & $3(10.0)$ & 1.000 \\
\hline Death, $n(\%)$ & $1(3.3)$ & $2(6.7)$ & 1.000 \\
\hline
\end{tabular}

Notes: ${ }^{a}$ median (interquartile range). Abbreviations: $R R H$ robotic radical hysterectomy, $L R H$ laparoscopic radical hysterectomy rectovaginal, vesicovagina and ureterovagina fistulas was noted in two surgical groups. Shorter indwelling time of bladder and drain catheters was observed in the RRH group than in the LRH group ( $p<0.05$ for all). Length of postoperative hospital stay in the RRH group was significantly shorter compared with that in the LRH group $(p<0.05)$. Patients in two surgical groups similarly experienced the recurrence and death. ( $p>0.05$ for all).

\section{Discussion}

Prevalence of cervical cancer has increased continuously over the last few decades in the entire globe, and ORH has been the most common surgical method in therapy of cervical cancer [4]. Subsequently, LRH has become the standard therapy of cervical cancer through providing the benefits of minimally invasive surgery $[5,6]$. Recently, RRH has revolutionized the therapy of cervical cancer, and its application has been in development. Compared with ORH, previous studies have found that RRH leads to significantly fewer complications and shorter length of hospital stay [7-10]. While LRH is widely accepted for the therapy of cervical cancer, the numbers of patients with RRH have not shown the same growth partly due to a lack of studies supporting a better role of RRH than LRH in therapy of cervical cancer [11-13]. There exists a need for prospective studies to provide the evidence whether the 
robot provides additional benefits for Chinese older women with cervical cancer $[14,15]$.

For the first time in China, this study directly compared RRH with LRH in Chinese older women with cervical cancer. In addition to shorter indwelling time of bladder and drain catheters, patients accepting the RRH had significantly fewer postoperative complications and shorter length of postoperative hospital stay compared with LRH in this study, suggesting a faster recovery benefiting from $\mathrm{RRH}$ rather than LRH. In these aspects, RRH is more favorable to the therapy of cervical cancer compared with LRH because robotic instrument provides superior safety by means of its three-dimensional viewing, comfortable fatigue-reducing console and improved motion freedom, which significantly reduces the ergonomic problems of conventional laparoscopic instrument [2, 3].

Surgical qualities and survival outcomes are significant aspects to observe when applying a new surgical method. A 4-center study with 23 patients by Tinelli $\mathrm{R}$ has compared survival outcomes of robotic method to that of laparoscopic method on radical hysterectomy, and realized no difference in numbers of recurrence between two groups during the follow-up [16]. However, numbers of death are not evaluated in above-mentioned study. There was no significant difference between RRH and LRH with respect to numbers of recurrence and death in this study. In regard to surgical qualities, there were not only no conversion to laparotomy, but also no severe complication happened in patients accepting the RRH. Compared with LRH, surgical specimens removed during the RRH had not only similar uterine size, tumor size, vaginal length and numbers of pelvic lymph nodes (metastasis), but also negative margins, supporting the efficacy and feasibility of robotic method to radical hysterectomy.

This study had some limitations. Firstly, this study focused on Chinese older women. It is therefore difficult to generalize the findings to women with cervical cancer of all ages. However, prospective studies comparing the efficacy and safety outcomes of RRH and LRH are scarce for older Chinese women. Secondly, this study applied an approach based on country-specific recommendations and therefore the findings cannot be extrapolated to women with cervical cancer worldwide. However, we feel that the findings are relevant to the clinical practice in China.

\section{Conclusions}

This study demonstrated that RRH provided additional benefits for Chinese older women with cervical cancer because of less complications and faster recovery when compared with LRH. Meanwhile, this study supported an equivalence of surgical qualities and survival outcomes of RRH to LRH. Robotics-assisted surgical method is effective, safe and feasible for Chinese older women with cervical cancer.

\section{Abbreviations}

FIGO: International federation of gynecology and obstetrics; LRH: Laparoscopic radical hysterectomy; ORH: Open radical hysterectomy; RRH: Robotic radical hysterectomy; SPSS: Statistic package for social science

\section{Acknowledgements}

We are grateful to all study participants for their participation in the study.

\section{Funding}

The authors have no funding to be reported.

\section{Availability of data and materials}

In attempt to preserve the privacy of patients, clinical data of patients will not be shared; data can be available from authors upon request.

\section{Authors' contributions}

$\mathrm{CL}$ conceived, designed and performed the study, analyzed the data, and wrote the paper. ML conceived, designed and performed the study, analyzed the data, and wrote the paper. XL conceived, designed and performed the study, analyzed the data, and wrote the paper. All authors read and approved the final manuscript.

\section{Ethics approval and consent to participate}

The study protocol was approved by Ethics Committee of Chinese People's Liberation Army General Hospital (Beijing, China). Each participant provided written informed consent to be included in the study.

\section{Consent for publication}

Not applicable.

\section{Competing interests}

The authors declare that they have no competing interests.

\section{Publisher's Note}

Springer Nature remains neutral with regard to jurisdictional claims in published maps and institutional affiliations.

\section{Author details}

'Department of Gynecology, Chinese People's Liberation Army General Hospital and Hainan Branch, Haitang Bay, Sanya, China. 'Department of Oncology, Chinese People's Liberation Army General Hospital and Hainan Branch, Sanya, China.

Received: 11 February 2017 Accepted: 16 March 2018

Published online: 01 May 2018

References

1. Nezhat FR, Datta MS, Liu C, Chuang L, Zakashansky K. Robotic radical hysterectomy versus total laparoscopic radical hysterectomy with pelvic lymphadenectomy fortreatment of early cervical cancer. JSLS. 2008;12:227-37.

2. Chen CC, Falcone T. Robotic gynecologic surgery: past, present, and future. Clin Obstet Gynecol. 2009;52:335-43.

3. Nezhat C, Lavie O, Lemyre M, Unal E, Nezhat CH, Nezhat F. Robot-assisted laparoscopic surgery in gynecology: scientific dream or reality? Fertil Steril. 2009;91:2620-2.

4. Quinn MA, Benedet IL, Odicino F, et al. Carcinoma of the cervix uteri. FIGO 26th annual report on the results of treatment in gynecological Cancer. Int J Gynecol Obstet. 2006;95:S43-S103.

5. Medeiros LR, Rosa DD, Bozzetti MC, et al. Laparoscopy versus laparotomy for benign ovarian tumour. Cochrane Database Syst Rev 2009:CD004751.

6. Nieboer TE, Johnson N, Lethaby A, et al. Surgical approach to hysterectomy for benign gynaecological disease. Cochrane Database Syst Rev 2009:CD003677.

7. Geisler JP, Orr CJ, Khurshid N, Phibbs G, Manahan KJ. Robotically assisted laparoscopic radical hysterectomy compared with open radical hysterectomy. Int J Gynecol Cancer. 2010;20:438-42.

8. Soliman PT, Frumovitz M, Sun CC, et al. Radical hysterectomy: a comparison of surgical approaches after adoption of robotic surgery in gynecologic oncol-ogy. Gynecol Oncol. 2011;123:333-6.

9. Spirtos NM, Eisenkop SM, Schlaerth JB, Ballon SC. Laparoscopic radical hysterectomy (type III) with aortic and pelvic lymphad-enectomy in patients 
with stage I cervical cancer: surgical morbidity and intermediate follow-up. Am J Obstet Gynecol. 2002;187:340-8.

10. Yim GW, Kim SW, Nam EJ, Kim YT. Role of robot-assisted surgery in cervical cancer. Int J Gynecol Cancer. 2011;21:173-81.

11. Kruijdenberg CB, van den Einden LC, Hendriks JC, Zusterzeel PL, Bekkers RL. Robot-assisted versus total laparoscopic radical hysterectomy in early cervical cancer, a review. Gynecol Oncol. 2011;120:334-9.

12. Advincula AP, Wang K. Evolving role and current state of robotics in minimally invasive gynecologic surgery. J Minim Invasive Gynecol. 2009;16:291-301.

13. Lin PS, Wakabayashi MT, Han ES. Role of robotic surgery in endometrial cancer. Curr Treat Options in Oncol. 2009;10:33-43.

14. Maggioni A, Minig L, Zanagnolo V, et al. Robotic approach for cervical cancer: comparison with laparotomy: a case control study. Gynecol Oncol. 2009;115:60-4

15. Boggess JF, Gehrig PA, Cantrell $L$, et al. A case-control study of robot-assisted type III radical hysterectomy with pelvic lymph node dissection compared with open radical hysterectomy. Am J Obstet Gynecol. 2008;199:357e1-7.

16. Tinelli $\mathrm{R}$, Malzoni $\mathrm{M}$, Cosentino $\mathrm{F}$, et al. Robotics versus laparoscopic radical hysterectomy with lymphadenectomy in patients with early cervical cancer: a multi-center study. Ann Surg Oncol. 2011;18:2622-8.

Submit your next manuscript to BioMed Central and we will help you at every step:

- We accept pre-submission inquiries

- Our selector tool helps you to find the most relevant journal

- We provide round the clock customer support

- Convenient online submission

- Thorough peer review

- Inclusion in PubMed and all major indexing services

- Maximum visibility for your research

Submit your manuscript at www.biomedcentral.com/submit 